\title{
Cytological Investigation of Pollen Development in Sorghum Line with Male Sterility Induced by Sodium Ascorbate in Tissue Culture
}

\author{
Marina I. Tsvetova, Lev A. Elkonin \\ Department of Biotechnology, Agricultural Research Institute for South-East Region, Saratov, Russia. \\ Email: lelkonin@gmail.com
}

Received May $15^{\text {th }}, 2013$; revised June $17^{\text {th }}, 2013$; accepted July $5^{\text {th }}, 2013$

Copyright (C) 2013 Marina I. Tsvetova, Lev A. Elkonin. This is an open access article distributed under the Creative Commons Attribution License, which permits unrestricted use, distribution, and reproduction in any medium, provided the original work is properly cited.

\begin{abstract}
Investigation of male sterility mutations is an effective approach for identification of genes involved in anther and pollen development. The comparison of "cytological phenotypes" of newly induced mutants with phenotypes determined by already known genes favors elucidation of genetic control of diverse microsporo- and gametogenesis stages. In this paper, we describe pollen development in the grain sorghum line Zh10-asc1 with mutation of male sterility. This line was obtained from callus culture treated by sodium ascorbate. A wide spectrum of abnormalities in microsporogenesis have been found, such as cytomixis, chromosomal laggards, chromosome disjunction, adhesion of chromosomes, disturbed cytokinesis, and others. In tapetum, the cells with one nucleus, with unequal nuclei, and with micronuclei have been observed. During pollen grain (PG) maturation abnormalities in starch accumulation and delay of development often took place. In mature anthers, a variety of pollen grain types have been revealed: fertile, of irregular shape, incompletely filled with starch, PGs delayed at the uni-nucleate or bi-nucleate gametophyte stages, with partially or fully degenerated contents, and with abnormal coloration. Variation in spectrum and the frequency of disturbances between the flowers of one and the same plant have been revealed. The reasons for significant genetic and epigenetic instability are discussed.
\end{abstract}

Keywords: Male Sterility; Microsporogenesis; Sodium Ascorbate; In Vitro Mutagenesis; Genetic Instability; Sorghum bicolor (L.) Moench

\section{Introduction}

Plant male sterility caused by spontaneous or experimentally induced mutations of nuclear or cytoplasmic genes is a wide area of research. Study of spontaneous and experimentally induced mutations allowed revealing a lot of mei-genes and genes controlling diverse stages of pollen formation [1-3]. Significant progress has been achieved in past years using T-DNA mutagenesis [4-7]. The majority of these studies were carried out using the model species such as Arabidopsis thaliana or Oryza sativa. For species which are more recalcitrant for genetic transformation, a possible approach consists in study of "cytological phenotypes" of new mutants and comparison of them with phenotypes of earlier investigated accessions with the identified genes. Investigation of "cytological phenotypes" of such mutants makes it possible to define temporal pattern of gene expression involved in male ga- mete formation and elucidate pollen developmental phases upon which the studied mutations affect.

Among factors inducing mutations of male sterility specific mutagens targeting plant mitochondrion is of special interest because of the significant role of mitochondrial genome in male sporo- and gametogenesis [8]. Previously, we have shown that treatment of sorghum callus cultures with some agents (streptomycin, ethidium bromide), which are known for their capacity to induce mutations of mitochondrial genes, allowed us to obtain the mutants with male sterility ( $m s-s t r, m s-b r 1)[9,10]$. In the present paper we report the results of cytological investigation of male sterility mutation, ms-asc 1 , which was found in sorghum plant regenerated from callus treated by sodium ascorbate. Sodium ascorbate is known to be one of the most efficient factors inducing lipid peroxidation in plant and animal mitochondria [11-13]; oxidizing 
stress occurring as a result of this process causes disturbances in functioning of mitochondrial genetic apparatus and damages of DNA [14-17]. The $m s$-asc1 mutation induced by sodium ascorbate, as well as $m s-s t r$ and $m s-b r 1$ mutations, is characterized by a broad spectrum of abnormalities of microsporogenesis, tapetum and pollen maturation that are described in this paper.

\section{Material and Methods}

\subsection{Plant Material}

The line Zh10-asc1 of the grain sorghum (Sorghum bicolor (L.) Moench) has been obtained following the treatment of the line Zheltozernoye-10 (Zh10) callus with sodium ascorbate [18]. One regenerated plant $\left(\mathrm{R}_{0}\right)$ from treated callus was characterized by complete male sterility. It was pollinated by the original line (Zh10), and fertile $\mathrm{F}_{1}$ hybrids have been obtained. In their progeny $\left(\mathrm{F}_{2}\right)$, semi-sterile plants (with $10 \%-20 \%$ seed set on the bagged panicles) have been found. Seed set was estimated as the ratio of florets with seeds to the total number of florets on the panicles bagged before beginning of anthesis and expressed as a percentage. The line Zh10-asc 1 resulted from self-pollination of such semi-sterile plants for three generations. Sterile, semi-sterile, and fertile plants from $\mathrm{F}_{4}\left(2009^{\text {th }}\right)$ and $\mathrm{F}_{5}\left(2010^{\text {th }}\right)$ were used for cytological investigation.

\subsection{Microscopy}

Panicle branches were fixed in 3:1 ethanol: acetic acid for $24 \mathrm{~h}$ and after double washing with $75 \%$ ethanol were stored at $4^{\circ} \mathrm{C}$. For investigation of meiosis, anthers extracted from flowers were treated with $\mathrm{HCl}(3.5 \%$ for 15 $\mathrm{min}$, then $50 \%$ for $20 \mathrm{~min}$ ), washed with distilled water for $2 \mathrm{~min}$, then they were treated with acetic acid (45\%) for $20-30 \mathrm{~min}$, and stained with acetohematoxiline (2\%) for 24 hours. Squashes were prepared using mixture of $45 \%$ acetic acid with $70 \%$ chloral-hydrate $(1: 1)$ colored with some drops of acetohematoxiline. Pollen grains (PGs) were stained with $1 \% \mathrm{I}_{2}-\mathrm{KI}$. Squashes for analyses of pollen were prepared from the anthers evoked from five individual branches from different parts of a panicle. PGs from each branch were estimated in four replications. Black pollen grains were presumed to be fertile.

\section{Results}

\subsection{First Division of Meiosis}

In the Zh10-asc1 plants, asynchrony of the meiotic processes took place in individual anthers: meiocytes at stages from prophase I up to tetrad were observed simultaneously.
At the premeiotic stage or at prophase I, cytomixisthe transfer of chromatin material from one cell to another-was observed in six out of nine plants studied (Figure 1(a)). It resulted in formation of microspore mother cells (MMCs) with extra chromatin masses or additional nuclei. In anthers of the cytomictic plants, the coenocytic structures, which arose, apparently, due to junction of two or more meiocytes were observed. In addition, binucleate cells with equal nuclei have been found. The nuclei in the binucleate cells and in the coenocytic structures were synchronized in their divisions (Figures 1(b) and (c)).

At diakinesis, the Zh10-asc1 plants had an increased frequency of MMCs with unpaired chromosomes $(12.0 \%$ - 23.5\%), in comparison with the plants of Zh10 $(0.0 \%$ $6.1 \%$ ). At telophase I, unpaired chromosomes and some of bivalents showed irregular behavior during chromosome disjunction and failed to disperse normally to the poles (Figure 1(d)). They were not included in the daughter nuclei, forming micronuclei or additional nuclei (Figure 1(e)). In the anaphase/telophase I, a lot of other aberrations associated with abnormal chromosome movement and cytokinesis disturbances were observed resulting in formation of monads and anomalous dyads (Figure 1(f)).

\subsection{Second Division of Meiosis}

Asynchronous division of dyad sister cells was not infrequent at the second division. During meta- and anaphase II the same types of irregularities that were found at the first division were observed: some chromosomes did not include in an equatorial plate; in telophase II, solitary or numerous chromosomes were scattered in the cytoplasm (Figure 1(g)) resulting in the tetrads with a lot of micronuclei (Figure 1(h)). Due to irregular cytokinesis triads were formed in some cases. In some MMCs, abnormalities associated both with chromosome disjunction and cytokinesis took place simultaneously resulting in formation of absolutely abnormal meiotic products (Figure $\mathbf{1}(\mathbf{m})$ ).

All the studied Zh10-asc1 plants had the dyads with the division spindles dislocated not in parallel, as is typical for the Poaceae species, but under different angles with respect to each other (Figure 1(i)). Correspondingly, the equatorial plates at metaphase II disposed abnormally (Figure 1(j)) that resulted in formation of tetrads with abnormal configuration (Figures 1(k) and (I)), their frequency ranging in different plants from $7.1 \%$ to $24.0 \%$.

Irregularities in chromosome disjunction led to the formation of microspores with numerous micronuclei.

Failure of cytokinesis led to coenocytic microspores formation, which often did not develop a normal cell 


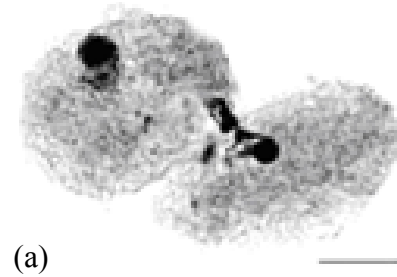

(a)

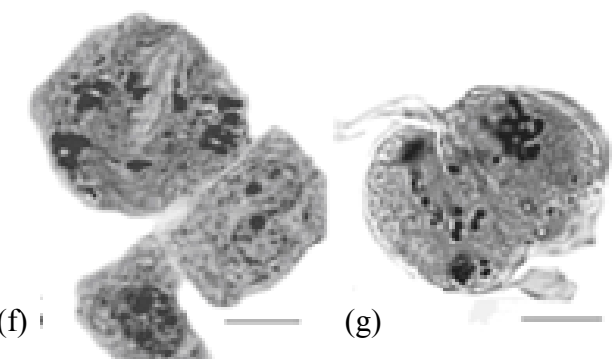

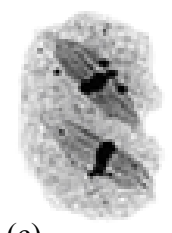

(c)

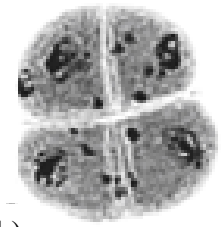

(h)

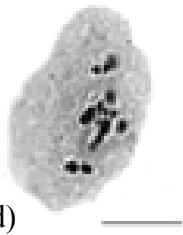

(d)

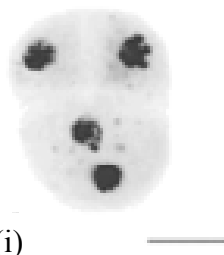

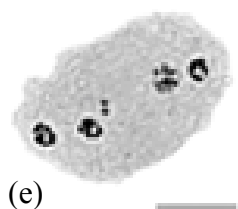

(j)

(i)

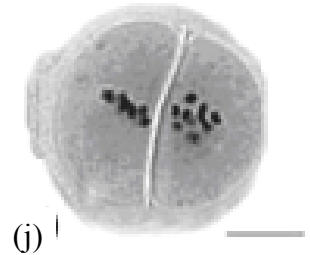

(k)!

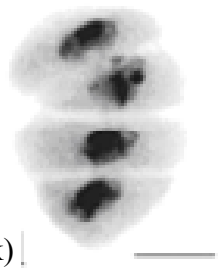

(1)

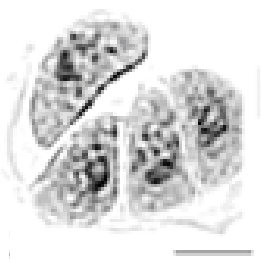

(m)

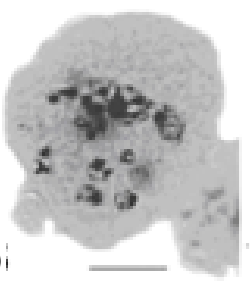

(n)

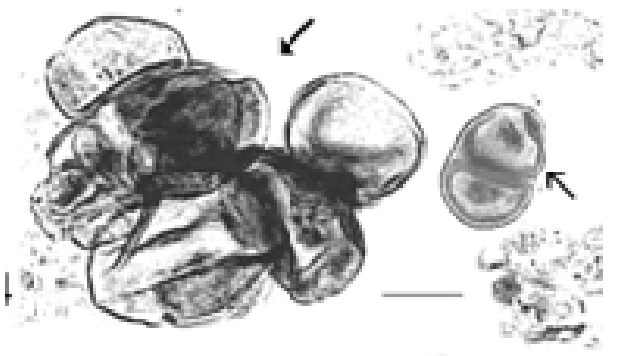

(r)

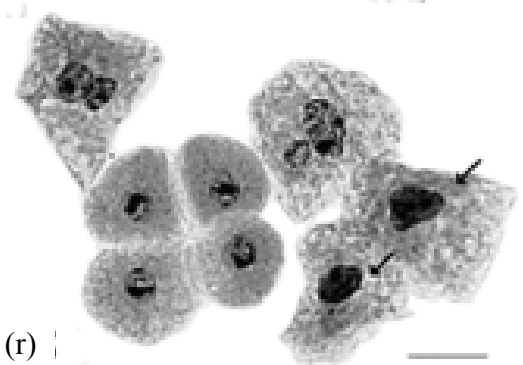

(o)

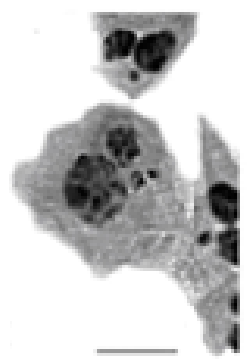

(p)

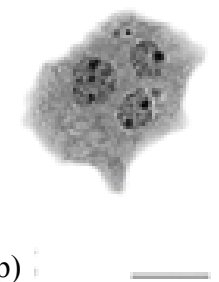

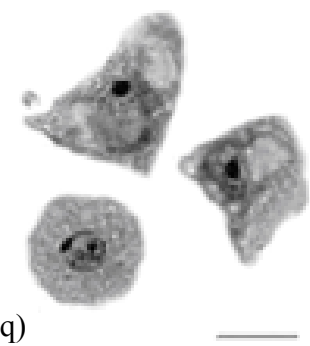

Figures 1. Abnormalities in pollen development in Zh10-asc1 plants. (a) Cytomixis; ((b) and (c)) First division in binucleate cells; (d) Scattering of chromosomes at telophase I; (e) Four-nucleate cell with micronuclei resulted from disturbed telophase I; (f) Dyad with numerous micronuclei and not completed cytokinesis, and tapetal cells; (g) Abnormalities in second meiotic division; (h) Tetrad with numerous micronuclei; ((i) and (j)) Diads with abnormal location of nuclei and equatorial plates occurred from anomalous disposition of division spindles during second meiotic division; ((k) and (l)) Tetrads having abnormal structure; (m) Abnormal product of the disturbed meiotic division; (n) Microspores developed from dyad cells ( $\rightarrow$ ) and microspores enclosed in common pollen wall $(\rightarrow)$; (o) Tapetal cells with unequal nuclei and micronuclei; (p) Tapetal cell with three nuclei; (q) Surplus vacuolization of tapetal cells; (r) Pycnosis in tapetal cells (arrows). All scale bars $=20 \mu \mathrm{m}$.

wall. In other cases, the second division was absent, and "the giant microspores", which synthesized pollen coat, were formed from the cells of dyads (Figure 1(m)).

The aberrations described above, along with cell degeneration, were found out in all the studied plants. But some aberrations were specific only to individual plants. For example, in the plant \#2-9 (F5), mitosis was found in microspores immediately after their release from the tetrads. In the plants \#6-7 and \#6-1 (both in F5), vacuolization of the microspores began before pollen grain coat and pore formation. In the same plants, some micro- spores did not form the pollen grain wall. In the plant \#2-4 (F5), the cells of dyads were covered by pollen coat (Figure 1(m)).

The frequencies of aberrations observed at microsporogenesis are summarized in the Table 1. Data for MMCs include all abnormalities found at the stages from metaphase I up to tetrad, with the dyads at all the stages of second meiotic division and the tetrads being considered as a one meiocyte.

The frequency of microsporogenesis irregularities in the Zh10-asc1 was higher and the limits of variation for 
Table 1. Frequency of aberrations at microsporogenesis and pollen fertility in Zh10-asc1 plants with different seed set.

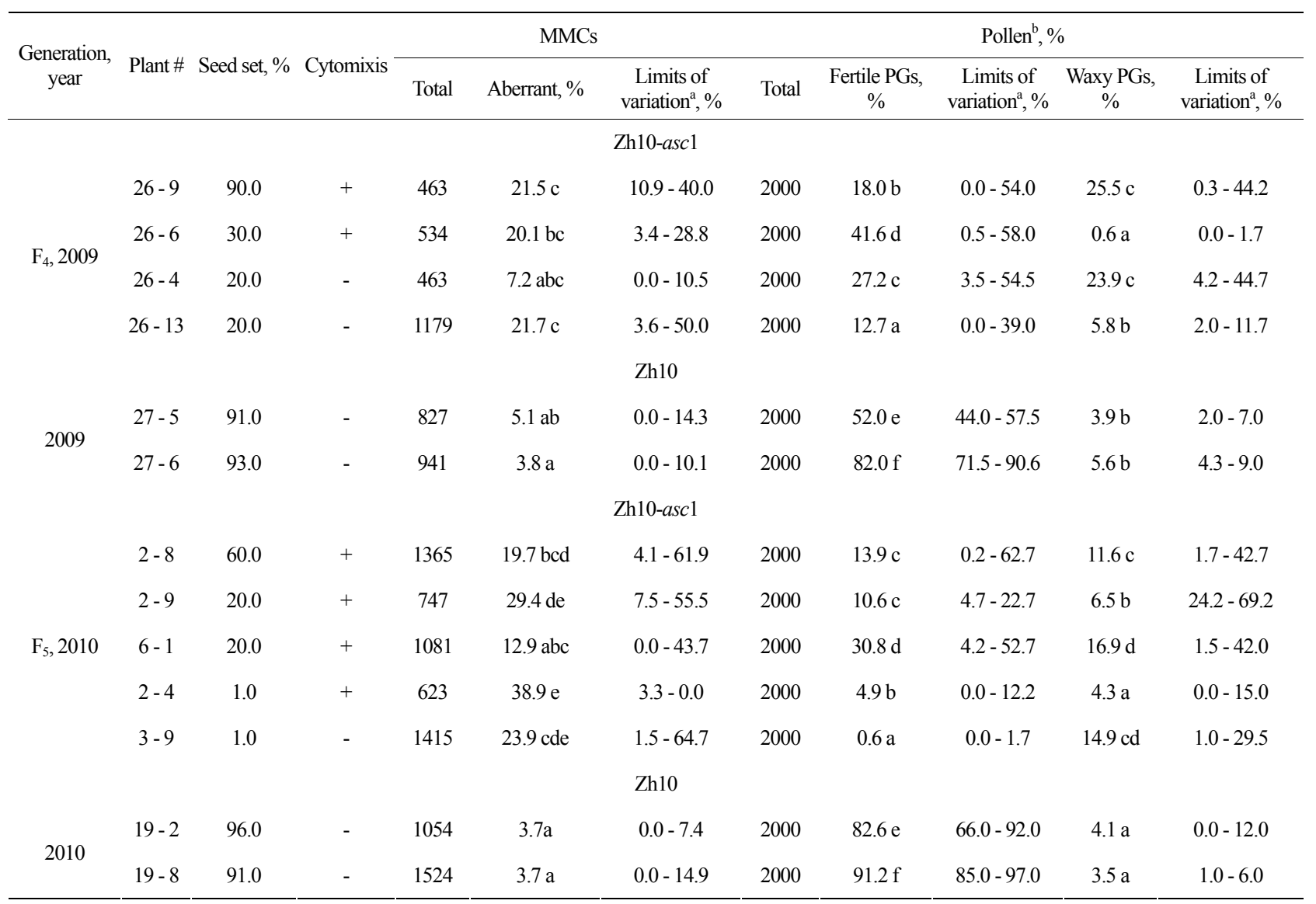

${ }^{a}$ Limits of variation represent data for different flowers (MMCs, dyads, and tetrads) and branches (pollen fertility); ${ }^{b}$ data for hermaphrodite flowers; Data followed by the same letter did not differ significantly within the same year at $p<0.05$ according to Duncan Multiple Range Test.

all the stages were considerably wider, in comparison with the original line Zh10 (Table 1), significant differences being observed not only between the plants, but also between the flowers of individual plant that hampered statistical evaluation of data. However, in spite of such wide variation, the plants of the line Zh10-asc1 with different seed set significantly differed in percentage of aberrations: the "almost" sterile plants (with 1\% seed set) (\#2 - 4 and \#3 - 9, (Table 1) had higher aberration frequency compared with semi-sterile plant \#2 - 8 (with $20 \%$ seed set). At the same time, semi-sterile plants \#2 9 and \#6 - 1 with the same seed set (20\%) also differed significantly in percentage of meiotic aberrations. No significant differences in the frequencies of aberrations have been found between the plants with cytomixis and without it.

\subsection{Tapetum}

In the fertile sorghum lines, at the leptotene stage in MMCs, mitosis without cytokinesis occurs in tapetum, resulting in cells with two nuclei of equal size. Nevertheless, a lit- tle proportion of mononuclear cells usually retains during the next stages of anther development [18]. In the Zh10asc 1 plants, mononuclear tapetal cells were more frequent than in the original line, $\mathrm{Zh} 10(8.1 \%-28.5 \%$ and $3.5 \%$ $3.9 \%$, respectively). In addition, the cells containing micronuclei or two nuclei differing in size have been revealed (Figure 1(o)), their frequency in Zh10-asc1 also being higher compared to Zh10 (2.5\% - 6.6\% vs. $1.2 \%$ $2.5 \%)$. In the same plants, three- and four-nucleated tapetal cells have been found (Figure 1(p)), which were not ever observed in the Zh10 line. This testifies that the plants of the Zh10-asc1 line parallel to meiotic abnormalities displayed partially disturbed mitosis in somatic tissues of anthers. In addition, in the plant \#2 - 4 (F5), the length of some tapetal cells reached $52.0 \mu \mathrm{m}$ whereas in other plants it did not exceed $40.5 \mu \mathrm{m}$. Besides that, in some flowers of this plant there were the cells with unusually highly developed vacuoles (Figure 1(q)). In all the plants, the tapetal cells with picnotic nuclei have been found (Figure 1(r)).

Different flowers of one and the same mutant plant exhibited different frequency of tapetal abnormalities, as 
it had been found for microsporogenesis (Table 2). For example, in the plant $\# 2-8$ the frequency of mononuclear cells ranged in different flowers from $2.0 \%$ to $36.0 \%$; in the plant $\# 2$ - 4 the frequency varied between $6.0 \%$ to $38 \%$, whereas in Zh10 plants the limits of variation were no more than $10 \%$.

\subsection{Mature Pollen}

Analysis of pollen at the flowering stage has shown a great variety of PG anomalies. Side by side with the fertile PGs, there were 1) the PGs of irregular shape filled with normally stained starch; 2) the PGs filled by starch not completely; 3) the PGs with brown or light tan color ("waxy"-type PGs); 4) the PGs with light grey color; 5) the PGs delayed at the mono-nucleate or bi-nucleate gametophyte stages; 6) the PGs with the normally formed pollen coat and pore, and partially or fully degenerated content (Figure 2). These types of PGs were found in the pollen of all the studied Zh10-asc1 plants.

It must be emphasized that pollen of different individual branches of one and the same plant differed in coloration that pointed out quantitative or qualitative differences in starch accumulation. For example, the plants \#2 8, \#2 - 4, and \#3 - 9 had the branches with normally filled "waxy" and fertile PGs, and the branches in which the most PGs were entirely degenerated (Figures 2(a), (b), (h) and (i)). Different branches of the panicle of the plant \#6-1 differed for the quality and the quantity of starch being accumulated (Figures 1(d) and (e)). Pollen from the same branch could also contain both fertile, and "waxy"-type PGs (Figure 1(f)) or both fertile and degenerated PGs.
The frequency of the other PG types varied considerably between the branches and the flowers of the one and the same plant. The percentage of fertile PGs in the plants of the line Zh10-asc1 was sufficiently lower than in the plants of Zh10 (Table 1).

It must be emphasized that in semi-sterile sorghum plants there were no anthers in a majority of the male flowers. However, in some male flowers anthers were observed, and sometimes they contained sufficiently higher proportion of fertile PGs in comparison with the hermaphrodite ones. For example, the male flowers of the F5 plant \#2 - 8 contained approximately $70 \%$ fertile PGs (Figure 2(c)), and, most probably, these fertile PGs provided relatively high seed set in this plant. Interestingly, cytomixis, most likely, was not the important factor, reducing pollen fertility: the cytomictic plants \#26 - 6 (F4) and \#6 - 1 (F5) had the highest pollen fertility among the plants of the line Zh10-asc1 (Table 1).

\section{Discussion}

The data presented above showed a lot of abnormalities in the processes of microspore and PG development in the Zh10-asc1 plants: 1) disturbed tapetal cell formation; 2) irregularities in microsporogenesis (cytomixis, aberrations associated with meiotic chromosome behavior, abnormal cytokinesis); 3) anomalies in starch accumulation in PGs. Each of these abnormalities may be the reason of pollen sterility in studied plants.

Meiotic irregularities usually result in microspores with unbalanced genome structure, which most frequently are non-viable. It is known that anomalies of chromosome segregation and cytokinesis are the result of the disturbances

Table 2. Frequency of anomalous tapetal cells in the line Zh10-asc1 of the grain sorghum (F5).

\begin{tabular}{|c|c|c|c|c|c|c|}
\hline \multirow{3}{*}{ Plant \# } & \multirow{3}{*}{ Seed set, \% } & \multirow{3}{*}{ Total } & \multicolumn{4}{|c|}{ Cell proportion } \\
\hline & & & \multicolumn{2}{|c|}{ Mononuclear } & \multicolumn{2}{|c|}{ With abnormal nuclei ${ }^{\mathrm{a}}$} \\
\hline & & & Mean, \% & Limits of variation, $\%$ & Mean, \% & Limits of variation, $\%$ \\
\hline \multicolumn{7}{|c|}{ Zh10-asc1 } \\
\hline $2-8$ & 60.0 & 800 & $10.5 \mathrm{a}$ & $2.0-36.0$ & 2.5 & $0.0-8.0$ \\
\hline $2-9$ & 20.0 & 600 & $28.5 \mathrm{c}$ & $17.0-43.0$ & 4.4 & $0.0-12.0$ \\
\hline $6-1$ & 20.0 & 1600 & $10.9 \mathrm{a}$ & $2.0-21.0$ & 6.6 & $0.0-13.0$ \\
\hline $2-4$ & 1.0 & 1100 & $20.6 \mathrm{~b}$ & $6.0-38.0$ & 5.4 & $0.0-15.0$ \\
\hline $3-9$ & 1.0 & 1400 & $8.1 \mathrm{a}$ & $1.0-23.0$ & 2.7 & $1.0-5.0$ \\
\hline \multicolumn{7}{|c|}{$\mathrm{Zh}-10$} \\
\hline $19-2$ & 96.0 & 800 & $3.9 \mathrm{a}$ & $2.0-10.0$ & 1.25 & $0.0-4.0$ \\
\hline $19-8$ & 91.0 & 800 & $3.5 \mathrm{a}$ & $0.0-8.0$ & 2.5 & $0.0-8.0$ \\
\hline
\end{tabular}

${ }^{a}$ includes cells with unequal nuclei and cells with micronuclei; Data followed by the same letter did not differ significantly within the same year at $p<0.05$ according to Duncan Multiple Range Test. 


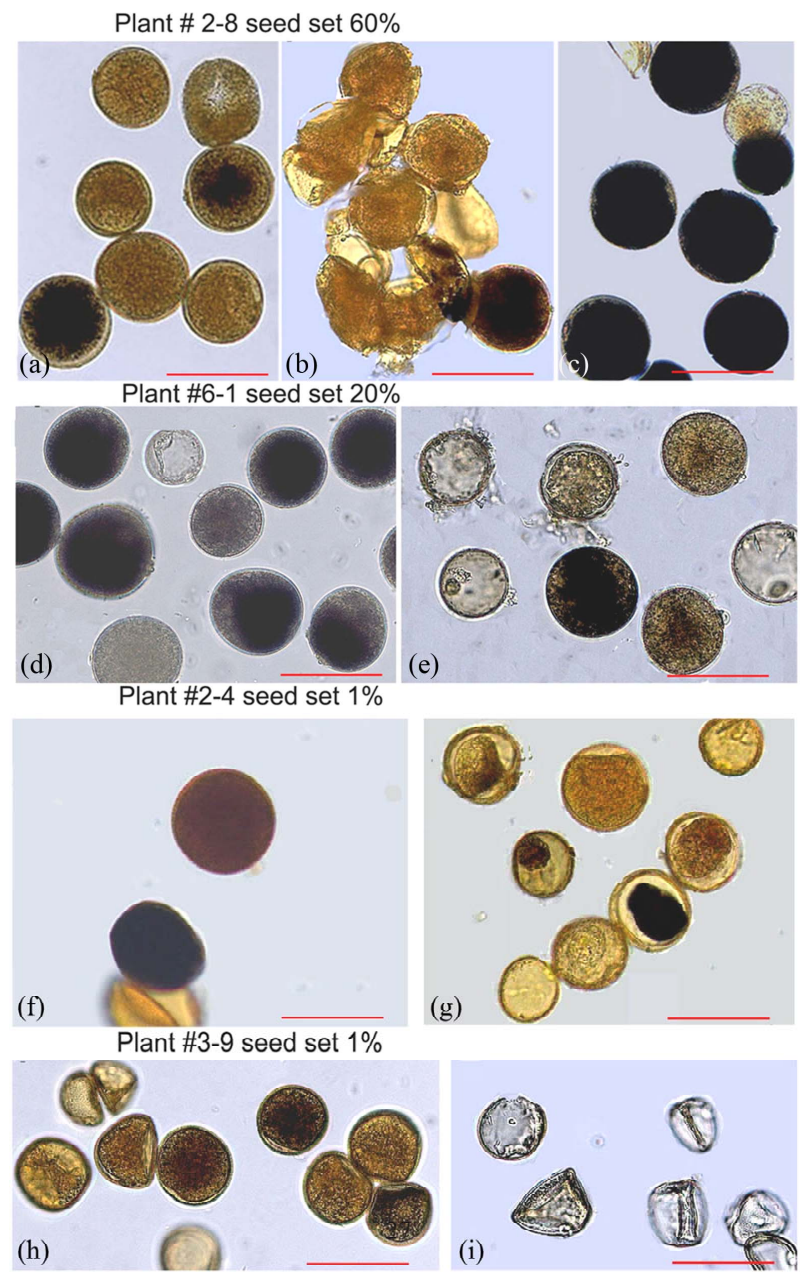

Figure 2. Pollen variation in different branches of the Zh10asc1 plants. ((a), (b)) "Waxy" and degenerated PGs from hermaphrodite flowers of the plant \#2 - 8. (c) Fertile PGs from the male flower of the plant \#2 - 8. ((d) and (e)) Pollen from "fertile" and "sterile" branches of plant \#6 - 1. ((f) and (g)) Pollen grains with different starch accumulation of the plant \#2 - 4. ((h), (i)) "Waxy" and completely degenerated pollen grains of the plant \#3-9. All scale bars $=50 \mu \mathrm{m}$.

of the genetic system controlling assembling and functioning of microtubules [19-21]. Whereas, the other abnormalities, have been found in Zh10-asc1 point to the mutations of the genes, which control other embryological processes. These processes are not caused by disturbances in the microtubular cell apparatus: disintegration of the tetrads, vacuolization and degeneration of the tapetal cells, accumulation of starch in the microspores, formation of the PG coat, etc. Each of these abnormalities resembles phenotypes described in previously identified mutants.

For example, mitosis occurred immediately after collapse of the tetrads of microspores (in the plant \#2 - 9) has been described in the maize plants possessing the recessive mutation polymitotic determining division in the microspores, bypassing the S-stage of the cell cycle [22]. The tetrad's failure to separate normally (the same plant \#2 - 9) is, more likely, connected with the mutation breaking pectin degradation that is necessary for microspore separation, as was shown in Arabidopsis [23,24]. Failure to form PG coat (the plant \#3 - 9) may be the result of the mutations disturbing sporopollenin synthesis [25]. Vacuolization of microspores before the formation of PG coat (in the plant \#26 - 1) has been previously described in maize plants with the mutation ms2 [26]. The disturbed orientation of two spindles in dyads completely coincides with the description of the mutation ms43 in maize [2].

A lot of abnormalities were observed also in the mature PGs (PG degeneration, changed coloration). PG degeneration could be caused by disturbed tapetum development. The tapetum of higher plants plays a critical role in pollen development, as it provides nutrition for developing microspores [27]. Disturbances in tapetum development (anomalous nuclei and increased vacuolization) found in the Zh10-asc1 anthers led, apparently, to disturbances of nutrition functions of this tissue that led in its turn to sterilization of a part of PGs. In addition, microspores that developed in abnormally configurated tetrads might lose contact with tapetal cells that also could result in disturbed nutrition of microspores.

Changes of PG coloration, obviously, are associated with abnormalities in accumulation and composition of starch. A variety of the PG coloration may point to instability in loci determining starch accumulation in PGs. The light tan, light- and dark-brown PGs may be caused by mutations in the waxy locus, which encodes granulebound starch synthase regulating amylose synthesis. Mutations in this locus result in amylose replacement by amylopectine [28]. Waxy genes were shown to be transposon-rich in a number of cereals [29]. Transposon insertions caused formation of endosperm sectors with null or intermediate $w x$ expression [30,31]. It is probable that in the Zh10-asc1 plants, the differences in pollen grain coloration were also determined by different functional states of waxy gene that might be caused also by transposon insertion(s). Indirect evidence in favor of this hypothesis is significant variation of PGs with different coloration in different branches of one and the same panicle. One of the reasons of such epigenetic variation as well as of high frequencies of other irregularities of pollen development in the Zh10-asc1 plants, each of which are known to be encoded by different genes, might be an active transposon insertion(s). Assuming that in similar highly variable male-sterile sorghum mutants ( $m s-b r 1)$ obtained by ethidium bromide treatment we found an active Isaac transposon [10], this hypothesis might be probable. 
Perhaps, both ethidium bromide and sodium ascorbate treatments having common target - the mitochondrial genetic system - might cause retrograde signal to nuclear genome resulting in activation of mobile genetic element(s).

Alternatively, broad spectrum of abnormalities in pollen development in Zh10-asc1 plants could be explained by mutation(s) in a gene encoding a transcription factor that regulates expression of other genes involved in pollen development. Presently, a number of transcription factors required for pollen development have been found and their role in male-sterile phenotype was shown [32-35].

Thus, for the first time, we have described male sterility mutation induced by sodium ascorbate that is characterized by a broad spectrum of abnormalities in microsporo- and gametogenesis. The nature of this genetic and epigenetic instability will be a subject of further investigations.

\section{Acknowledgements}

This work was supported partly by the Russian Foundation for Basic Research (project no. 13-04-01404).

\section{REFERENCES}

[1] K. Kitada, N. Kurata, H. Satoh and T. Omura, "Genetic Control of Meiosis in Rice, Oriza sativa L. Classification of Meiotic Mutants Induced by MNU and Their Cytogenetical Characteristics," Japan Journal of Genetics, Vol. 58, No. 3, 1983, pp. 231-240. doi:10.1266/jjg.58.231

[2] I. N. Golubovskaya, "Meiosis in Maize: Mei Genes and Conception of Genetic Control of Meiosis," Advances in Genetics, Vol. 26, 1989, pp. 149-192. doi:10.1016/S0065-2660(08)60225-4

[3] W. Z. Cande, I. N. Golubovskaya, C. J. R. Wang and L. Harper, "Meiotic Genes and Meiosis in Maize," In: J. L. Bennetzen and S. Hake, Eds., Handbook of Maize, Vol. II, 2009, pp. 353-375. doi:10.1007/978-0-387-77863-1 18

[4] A. P. Caryll, H. J. Gareth and F. C. H. Franklin, "Dissecting Plant Meiosis Using Arabidopsis thaliana Mutants," Journal of Experimental Botany, Vol. 54, Plant Reproduction Biology, Special Issue, 2003, pp. 25-38.

[5] E. V. Deineko, A. A. Zagorskaya and V. K. Shumny, "TDNA Induced Mutants in Transgenic Plants," Russian Journal of Genetics, Vol. 43, No. 1, 2007, pp. 5-17. doi:10.1134/S1022795407010012

[6] M. Borg, L. Brownfield and D. Twell, "Male Gametophyte Development: A Molecular Perspective," Journal of Experimental Botany, Vol. 60, No. 5, 2009, pp. 1465-1478. doi:10.1093/jxb/ern355

[7] Z. A. Wilson and D. B. Zhang, "From Arabidopsis to Rice, Pathways in Pollen Development," Journal of Experimental Botany, Vol. 60, No. 5, 2009, pp. 1479-1492. doi:10.1093/jxb/erp095

[8] S. Fujii and K. Toriyama, "Genome Barriers between Nu- clei and Mitochondria Exemplified by Cytoplasmic Male Sterility," Plant Cell Physiology, Vol. 49, No. 10, 2008, pp. 1484-1494. doi:10.1093/pcp/pcn102

[9] L. A. Elkonin and M. I. Tsvetova, "Genetic and Cytological Analyses of the Male Sterility Mutation Induced in a Sorghum Tissue Culture with Streptomycin," Russian Journal of Genetics, Vol. 44, No. 5, 2008, pp. 575-583. doi:10.1134/S1022795408050104

[10] L. A. Elkonin, G. A. Geraschenkov, M. I. Tsvetova and N. A. Rozhnova, "Genetic Variation in a Sorghum Line with Multiple Genetic Instability Induced with Ethidium Bromide in an in Vitro Culture," Russian Journal of Genetics, Vol. 46, No. 7, 2010, pp. 805-815. doi:10.1134/S1022795410070057

[11] V. K. Voinikov, Yu. M. Konstantinov and V. I. Negruk, "Genetic Functions of Plant Mitochondria," Nauka, Sib. Branch, Novosibirsk, 1991.

[12] R. Milczarek, J. Klimek and L. Zelewski, "The Effects of Ascorbate and Alpha-Tocopherol on the NADPH-Dependent Lipid Peroxidation in Human Placental Mitochondria," Molecular Cell Biochemistry, Vol. 210, No. 1-2, 2000, pp. 65-73. doi:10.1023/A:1007007213846

[13] T. Sen, N. Sen, G. Tripathi, U. Chatterjee and S. Chakrabarti, "Lipid Peroxidation Associated Cardiolipin Loss and Membrane Depolarization in Rat Brain Mitochondria," Neurochemistry International, Vol. 49, No. 1, 2006, pp. 20-27. doi:10.1016/j.neuint.2005.12.018

[14] J. H. Song, S. H. Shin, W. Wang and G. M. Ross, “Oxidative Stress Induced by Ascorbate Causes Neuronal Damage in an in Vitro System," Brain Research, Vol. 895, No. 1-2, 2001, pp. 66-72. doi:10.1016/S0006-8993(01)02029-7

[15] C. Wan, S. Li, L. Wen, J. Kong, K. Wang and Y. Zhu, "Damage of Oxidative Stress on Mitochondria during Microspores Development in Honglian CMS Line of Rice," Plant Cell Reports, Vol. 26, No. 3, 2007, pp. 373 382. doi:10.1007/s00299-006-0234-2

[16] A. T. Hoye, J. Davoren, P. Wipf, M. P. Fink and V. E. Kagan, "Targeting mitochondria," Accounts of Chemical Research, Vol. 41, No. 1, 2008, pp. 87-97. doi:10.1021/ar700135m

[17] S. S. Gill and N. Tuteja, "Reactive Oxygen Species and Antioxidant Machinery in Abiotic Stress Tolerance in Crop Plants," Plant Physiology and Biochemistry, Vol. 48, No. 12, 2010, pp. 909-930. doi:10.1016/j.plaphy.2010.08.016

[18] M. I. Tsvetova, L. A. Elkonin and O. V. Ukolova, "Investigation of Tapetal Cells by Means of Squash Technique," Botanicheskiy Zhurnal, Vol. 91, No. 7, 2006, pp. 969974.

[19] R. K. Dawe, "Meiotic Chromosome Organization and Segregation in Plants," Annual Review of Plant Physiology. Plant Molecular Biology, Vol. 49, 1998, pp. 371-395. doi:10.1146/annurev.arplant.49.1.371

[20] A. M. Bhatt, C. Listen, T. Page, P. Fransz, K. Findlay, G. H. Jones, H. G. Dickinson and C. Dean, "The DIF1 Gene of Arabidopsis Is Required for Meiotic Chromosome Se- 
gregation and Belongs to the REC8/RAD21 Cohesion Gene Family," Plant Journal, Vol. 19, No. 4, 1999, pp. 463-472. doi:10.1046/j.1365-313X.1999.00548.x

[21] N. V. Shamina, "Diagnosticum of Abnormalities of Plant Meiotic Divisions," Tsitologia, Vol. 48, No. 6, 2006, pp. 486-494.

[22] K. W. Wolfe and Q. Liu, "The Maize Mutant polymitotic Affects Cell Cycle Events during Microspore Development," Planta, Vol. 210, No. 1, 1999, pp. 27-33. doi: $10.1007 / \mathrm{s} 004250050650$

[23] S. Y. Rhee and C. R. Somerville, "Tetrad Pollen Formation in quartet Mutants of Arabidopsis thaliana Is Associated with Persistence of Pectic Polysaccharides of the Pollen Mother Cell Wall," Plant Journal, Vol. 15, No. 1, 1998, pp. 79-88. doi:10.1046/j.1365-313X.1998.00183.x

[24] S. Y. Rhee, E. Osborne, P. D. Poindexter and C. R. Somerville, "Microspore Separation in the quartet $3 \mathrm{Mu}-$ tants of Arabidopsis Is Impaired by a Defect in a Developmentally Regulated Polygalacturonase Required for Pollen Mother Cell Wall Degradation," Plant Physiology, Vol. 133, No. 3, 2003, pp. 1170-1180. doi:10.1104/pp.103.028266

[25] T. Ariizumi and K. Toriyama, "Genetic Regulation of Sporopollenin Synthesis and Pollen Exine Development," Annual Review of Plant Biology, Vol. 62, 2011, pp. 437460. doi:10.1146/annurev-arplant-042809-112312

[26] M. C. Albersten and R. L. Phillips, "Developmental Cytology of 13 Genetic Male Sterile Locy in Maize," Canadian Journal of Genetics and Cytology, Vol. 23, No. 2, 1981, pp. 195-208.

[27] P. Bedinger, "The Remarkable Biology of Pollen," Plant Cell, Vol. 4, No. 8, 1992, pp. 879-887.

[28] J. F. Pedersen, S. R. Bean, D. L. Funell and R. A. Graybosch, "Rapid Iodine Staining Techniques for Identifying the Waxy Phenotype in Sorghum Grain and Waxy Genotype in Sorghum Pollen," Crop Science, Vol. 44, No. 3, 2004, pp. 764-767.

[29] J. F. Pedersen, S. R. Bean, R. A. Graybosch, S. H. Park and M. Tilley, "Characterization of Waxy Grain Sorghum Lines in Relation to Granule-Bound Starch Synthase," Euphytica, Vol. 144, No. 1-2, 2005, pp. 151-156. doi:10.1007/s10681-005-5298-5

[30] B. K. McClintock, "Further Studies of Gene-Control Systems in Maize," Carnegie Institute of Washington. Yearbook, Vol. 62, 1963, pp. 486-493.

[31] C. F. Weil, S. Marillonnet, B. Burr and S. R. Wessler, "Change in State $W x-m 5$ Allele of Maize Are Due to Intragenic Transposition of Ds," Genetics, Vol. 130, No. 1, 1992, pp. 175-185.

[32] T. Ito, N. Nagata, Y. Yoshiba, M. Ohme-Takaqi, H. Ma and K. Shinozaki, "Arabidopsis Male Sterility1 Encodes a PHD-Type Transcription Factor and Regulates Pollen and Tapetum Development," Plant Cell, Vol. 19, No. 11, 2007, pp. 3549-3562. doi:10.1105/tpc.107.054536

[33] C. Yang, G. Vizcay-Barrena, K. Conner and Z. A. Wilson, "Male Sterility1 Is Required for Tapetal Development and Pollen Wall Biosynthesis," Plant Cell, Vol. 19, No. 11, 2007, pp. 3530-3548. doi:10.1105/tpc.107.054981

[34] J. Zhu, G. Zhang, Y. Chang, X. Li, J. Yang, X. Huang, Q. $\mathrm{Yu}, \mathrm{H}$. Chen, T. Wu and Z. Yang, "AtMYB103 Is a Crucial Regulator of Several Pathways Affecting Arabidopsis Anther Development," Science China. Life Science, Vol. 53, No. 9, 2010, pp. 1112-1122.

[35] H. A. Phan, S. Iacuone, S. F. Li and R. W. Parish, "The MYB80 Transcription Factor Is Required for Pollen Development and the Regulation of Tapetal Programmed Cell Death in Arabidopsis thaliana," Plant Cell, Vol. 23 , No. 6, 2011, pp. 2209-2224. doi:10.1105/tpc.110.082651 